\title{
Value-based Healthcare
}

\section{Value-based Healthcare: The Value of Considering Patient Preferences and Circumstances in Orthopaedic Surgery}

\author{
David Ring MD, PhD, Kevin J. Bozic MD, MBA
}

$\mathrm{F}$ rom the Column Editor:

The name of my column has changed from "Orthopaedic Healthcare Worldwide" to "Valuebased Healthcare" to reflect the shift in our healthcare payment and delivery system, which incentivizes and rewards "value" (outcomes that matter to

A Note from the Editor-in-Chief: We are pleased to present to readers of Clinical Orthopaedics and Related Research ${ }^{\mathbb{R}}$ the latest Value-based Healthcare column (formerly Orthopaedic Healthcare

Worldwide). Value-based Healthcare explores strategies to enhance the value of musculoskeletal care by improving health outcomes and reducing the overall cost of care delivery. We welcome reader feedback on all of our columns and articles; please send your commentstoeic@clinorthop.org.

The authors certify that they, or any members of their immediate families, have no funding or commercial associations (eg, consultancies, stock ownership, equity interest, patent/ licensing arrangements, etc.) that might pose a conflict of interest in connection with the submitted article.

All ICMJE Conflict of Interest Forms for authors and Clinical Orthopaedics and Related Research ${ }^{\circledR}$ editors and board members are on file with the publication and can be viewed on request.

The opinions expressed are those of the writers, and do not reflect the opinion or policy of $C O R R^{\circledR}$ or The Association of Bone and Joint Surgeons ${ }^{\circledR}$. patients/cost to achieve those outcomes) over volume and intensity of services provided. The Value-based Healthcare column will highlight novel strategies from across the United States, and throughout the world, to enhance the value of care we provide to our patients, and improve the health of the populations we treat. We hope you enjoy reading this column as much as I enjoy preparing it, and I look forward to your continued feedback.

- Kevin J. Bozic MD, MBA

$$
* * *
$$

From the patient's perspective, seeking value in healthcare is not new, but a more-specific definition of "value" is gaining acceptance: Health outcomes achieved per dollar spent to achieve those outcomes [3]. Health

D. Ring $\mathrm{MD}, \mathrm{PhD}$

Hand and Upper Extremity Service, Department of Orthopaedic Surgery, Massachusetts General Hospital, Boston, MA, USA

\section{K. J. Bozic MD, MBA ( $\square)$}

Department of Surgery and Perioperative Care, Dell Medical School at the

University of Texas at Austin, 1400

Barbara Jordan Blvd., Suite 1.114,

Austin, TX 78723, USA

e-mail: kevin.bozic@austin.utexas.edu outcomes of interest to our patients include both the quality of care delivered and the experience patients and their families go through when receiving their care. If two medications provide the same health benefit, the less-expensive one provides greater value. In reality, though, the situation often is more complex and nuanced. For instance, a surgical procedure done as an outpatient or with a less expensive implant might seem to have greater value at the outset, but the value is reduced if there problems develop down the line like more unplanned readmissions or reoperations, more adverse events, or premature failure of the implant.

Quality currently is measured largely in terms of structure (professional certification, nurse-to-bed ratios) and process (site marking, administration of preoperative antibiotics), perhaps because these are more readily quantified and tracked. But the ideal quality measures address outcomes of specific interest to patients such as mortality, adverse events, unplanned readmission or reoperation, patient satisfaction, symptom intensity, and magnitude of limitations-the factors that structure and process measures are trying to 
improve upon [2]. In orthopaedic surgery, the most important outcomes from a patient's perspective, are improvement in quality of life or function, and reduction in pain.

When considering quality and value, it is important to distinguish preferencesensitive from nonpreference-sensitive medical conditions. A person severely dehydrated from Cholera may die without intravenous fluids. A person with HIV infection will develop AIDS and die without antiretroviral therapy. Necrotizing fasciitis and compartment syndrome are limb- and life-threatening without surgery. These conditions are not preference-sensitive: The correct treatment is often clear on diagnosis. In contrast, people with severe hip arthritis, large rotator cuff defects, or advanced carpal tunnel syndrome may prefer to avoid surgery. These conditions are preference-sensitive: Some people prefer to adapt to impairments from these conditions rather than face the risks, discomforts, and inconveniences of surgery. A substantial proportion of musculoskeletal conditions are preference-sensitive.

Given that preference-sensitive musculoskeletal conditions largely affect quality of life, the best outcome measures may be those that address symptoms, limitations, and satisfaction with care. These outcomes are referred to as patient reported outcomes (PROs) and they are measured using patient reported outcome measures (PROMs). It is worth reflecting on the potential of PROMs to transform the care of musculoskeletal illness. Orthopaedic surgeons expect fewer symptoms and limitations when surgery improves motion, when radiographic arthritis is less severe, and when a sutured rotator cuff defect heals. We are surprised to find that greater motion, more severe radiographic arthritis, and persistent defects after surgery are not always associated with reductions in symptoms and functional limitations [4]. We are puzzled by the ability of sham surgery and injection to relieve symptoms [5]. It is curious. It goes against our training and expertise.

With more routine use of PROMs, surgeons will want to pay more attention to the factors that have the strongest influence on these scores: Stress, distress, and ineffective coping strategies [4]. The influence of mindset and circumstances on musculoskeletal illness is evident to orthopedic surgeons of all subspecialties. But we have tended to limit our attention largely to objective pathophysiology and impairment, placing less emphasis on a patient's thoughts, emotions, and behaviors in response to their disease. In other words, we have tended to operate within the biomedical model where every illness can be reduced to a specific pathophys- iology. Improving quality and value in orthopaedic care is likely to benefit from considering each patient's illness from a biopsychosocial perspective.

When a patient seems to have more symptoms and more limitations than one would expect for a given pathophysiology, consider why this might be. Some patients with preference-sensitive conditions are dissatisfied with nonoperative treatment. For conditions that are expected with age - where it is possible that a substantial subset of people never seek care and are satisfied with their symptoms and impairments (trapeziometacarpal arthrosis or rotator cuff tendinopathy, for example)—it might help to ask why some patients are dissatisfied, and what can be done to help them become more satisfied. Interventions to minimize stress and distress and encourage effective coping strategies may achieve desired health outcomes on their own, and they are likely improve the results of our surgical interventions as well. Orthopaedic interventions may have their greatest value when patients in the best possible frame of mind and circumstances follow their preferences and take an active role in their health [1].

\section{References}

1. Andrawis J, Akhavan S, Chan V, Lehil M, Pong D, Bozic KJ. Higher preoperative patient activation associated with 


\section{Value-based Healthcare}

better patient-reported outcomes after total joint arthroplasty. Clin Orthop Relat Res. 2015;473:2688-2697.

2. National Quality Measures Clearinghouse. Selecting health outcome measures for clinical quality measurement. Available at: http://www.quality measures.ahrq.gov/tutorial/HealthOut
comeMeasure.aspx. Accessed October 18, 2015.

3. Porter ME. Competitive Advantage: Creating and Sustaining Superior Performance. New York, NY: Simon \& Schuster Inc; 2008.

4. Vranceanu AM, Barsky A, Ring D. Psychosocial aspects of disabling musculoskeletal pain. J Bone Joint Surg Am. 2009;91:2014-2018.

5. Wartolowska K, Judge A, Hopewell S, Collins GS, Dean BJ, Rombach I, Brindley D, Savulescu J, Beard DJ, Carr AJ. Use of placebo controls in the evaluation of surgery: systematic review. BMJ. 2014;348:g3253. 